\title{
ARTICLE
}

\section{Nanometric holograms based on a topological insulator material}

Zengji Yue ${ }^{1, \star}$, Gaolei Xue $e^{1,2, \star}$, Juan Liu ${ }^{2}$, Yongtian Wang ${ }^{2} \&$ Min Gu${ }^{1}$

Holography has extremely extensive applications in conventional optical instruments spanning optical microscopy and imaging, three-dimensional displays and metrology. To integrate holography with modern low-dimensional electronic devices, holograms need to be thinned to a nanometric scale. However, to keep a pronounced phase shift modulation, the thickness of holograms has been generally limited to the optical wavelength scale, which hinders their integration with ultrathin electronic devices. Here, we break this limit and achieve $60 \mathrm{~nm}$ holograms using a topological insulator material. We discover that nanometric topological insulator thin films act as an intrinsic optical resonant cavity due to the unequal refractive indices in their metallic surfaces and bulk. The resonant cavity leads to enhancement of phase shifts and thus the holographic imaging. Our work paves a way towards integrating holography with flat electronic devices for optical imaging, data storage and information security.

\footnotetext{
${ }^{1}$ Laboratory of Artificial-Intelligence Nanophotonics and CUDOS (Centre for Ultrahigh bandwidth Devices for Optical Systems), School of Science, RMIT University, Melbourne, Victoria 3001, Australia. ${ }^{2}$ Beijing Engineering Research Centre for Mixed Reality and Advanced Display, School of Optoelectronics, Beijing Institute of Technology, Beijing 100081, China. * These authors contributed equally to this work. Correspondence and requests for materials should be addressed to M.G. (email: min.gu@rmit.edu.au).
} 
olography has been widely used in optical microscopy, imaging, data storage, metrology as well as information security since it was invented by Dennis Gabor in 1940s (refs 1-3). The rapid developments of laser and computer technologies leaded to the advent of computer-generated holography (CGH) in 1960s (ref. 4). CGH holds a distinct superiority over the conventional optical holograms because real objects are not required for holographic imaging. It enables generating holographic interference patterns of virtual objects by digitally computing optical wavefronts ${ }^{5}$. With this advantage, $\mathrm{CGH}$ has a great potential to be integrated with modern electronic devices, such as smartphone and smart watch for imaging and display ${ }^{6}$. To realize these devices, holograms are required to be thinned to a nanometre scale. However, conventional holograms modulate the phase of light based on the propagation retardation in three-dimensional (3D) bulky materials. The phase modulation capability relies on the propagating distance through the materials, which requires the thickness of holograms within optical wavelength ranges. This drawback impedes the development of nanometric holograms and their integration with modern electronic devices.

The recent development of emerging flat optics demonstrates the potential to break the thickness limit ${ }^{7-9}$. Ultrathin holograms have been designed using various metasurfaces that consist of nanostructure arrays with alterable geometric parameters ${ }^{3,10-13}$. However, limited by the fundamental mechanism of Mie resonances (scattering), dielectric metasurface holograms are unlikely to reach a deep sub-wavelength scale (much less than the optical wavelength). Moreover, the nanofabrication of metasurface holograms requires quite complex facilities, such as focused ion beam lithography and electron-beam lithography. In addition, using the complex focused ion beam lithography or electron-beam lithography techniques, it is also hard to realize large area holograms for practical applications.

Two-dimensional (2D) materials that have attracted a great attention nowadays can provide an excellent platform for realizing ultrathin optical devices ${ }^{14,15}$. Topological insulators are unique quantum materials that have topologically protected $1 \mathrm{D}$ edge states on $2 \mathrm{D}$ insulating bulk states, or $2 \mathrm{D}$ metallic surface states on $3 \mathrm{D}$ insulating bulk states ${ }^{16,17}$. They demonstrate a number of fascinating electronic and optical properties, such as spinmomentum locking, near-infrared transparency and ultrabroadband plasmon excitations ${ }^{18-20}$. It has also been discovered that topological insulator materials hold the low refractive index in the surface layer but the ultrahigh refractive index in the bulk ${ }^{21}$. This unique property makes them promising for designing novel optoelectronic devices including nanometric holograms.

Here, we propose the concept and the experimental realization of nanometric holograms using a topological insulator material $\mathrm{Sb}_{2} \mathrm{Te}_{3}$. We observe that the $\mathrm{Sb}_{2} \mathrm{Te}_{3}$ thin film holds unequal refractive indices in the surface layer and bulk, which makes the thin film acting as an intrinsic resonant cavity. The optical path length and phase shifts of an output light beam from the cavity can be enhanced through internal multi-reflections. Based on this new mechanism, we achieve $60 \mathrm{~nm}$ binary holograms of $3 \mathrm{~mm} \times 3 \mathrm{~mm}$ in size using a simple and fast direct laser writing (DLW) system ${ }^{2}$. Holographic images are successfully realized with the nanometric holograms.

\section{Results}

Design of nanometric holograms. The conceptual design of the nanometric hologram using the $\mathrm{Sb}_{2} \mathrm{Te}_{3}$ thin film is illustrated in Fig. 1. First, a binary phase-only hologram of the object is calculated by a computer (Supplementary Fig. 1). Then we record the binary hologram into the $\mathrm{Sb}_{2} \mathrm{Te}_{3}$ thin film through programmed multifocal spots created by the Debye diffraction method $^{22,23}$ as shown in Fig. 1a. A home-built DLW system is used for the binary hologram fabrication (see Methods section). The binary holograms of the object images are first converted into a series of phase diagrams, which are uploaded onto the spatial light modulator in certain time orders. Then the binary holograms are printed into the $\mathrm{Sb}_{2} \mathrm{Te}_{3}$ thin film through synchronously moving the sample stage in the $x y$-plane. The number of focal-spots of the laser beam is 60 by 60 and the diameter of each spot is $2 \mu \mathrm{m}$. The size of the fabricated hologram is $3 \times 3 \mathrm{~mm}^{2}$ and the number of pixels is $1,500 \times 1,500$. The laser beam fluence is kept at $0.1 \mathrm{~J} \mathrm{~cm}^{-2}$.

The upper inset in Fig. 1a displays two pixels ablated by the focused laser spots in the $\mathrm{Sb}_{2} \mathrm{Te}_{3}$ thin film. The area of the thin film without ablation leads to an enhanced phase shift due to the resonant cavity inside it. Based on this principle, a phase-only binary hologram is realized. Figure $1 b$ shows the holographic imaging procedure by using a continuous-wave laser beam. Holographic images are reconstructed by illuminating the $\mathrm{Sb}_{2} \mathrm{Te}_{3}$ thin film with a laser beam and are captured by a colour CCD camera. The projection angle $\theta$ of the holographic image is designed to avoid the overlapping between the holographic image and the zero-order beam.

Analysis of the multilayer structure. The multilayer structure of the $\mathrm{Sb}_{2} \mathrm{Te}_{3}$ thin film on a $\mathrm{Si}$ substrate is schematically shown in Fig. 2a. The dielectric bulk of the $\mathrm{Sb}_{2} \mathrm{Te}_{3}$ thin film is sandwiched within the two metallic surface layers. The refractive index $(n)$ and extinction coefficient $(k)$ of the surface layers and the bulk of the $\mathrm{Sb}_{2} \mathrm{Te}_{3}$ thin film are measured by using a spectroscopic ellipsometer (see Methods section). The measured wavelengths range from 350 to $1,000 \mathrm{~nm}$. The measured data are fitted by using a B-spline model for the surface layers and a Tauc-Lorentz model for the bulk. The details of these models can be found in our previous work ${ }^{21}$.

Figure $2 \mathrm{~b}$ shows the $n$ and $k$ curves of the surface layers of the $\mathrm{Sb}_{2} \mathrm{Te}_{3}$ thin film. Figure $2 \mathrm{c}$ shows the $n$ and $k$ curves of the bulk of the film. The value of bulk $n$ agrees well with the previously reported values elsewhere ${ }^{24}$. Both $n$ and $k$ values are unequal in the bulk and the surface layers from visible to near-infrared wavelength ranges. With the unequal refractive indices, an $\mathrm{Sb}_{2} \mathrm{Te}_{3}$ thin film acts as an intrinsic optical resonant cavity. Two surface layers serve as two reflectors. The bulk behaves as an optical resonant cavity. Thus an incident light beam can be reflected multiple times between two surface layers and partially be confined in the bulk. The phase modulation of the reflected light beam from the resonant cavity can be enhanced.

The COMSOL Multiphysics modelling software is used to simulate the reflection and phase shift of the output light beams from the $\mathrm{Sb}_{2} \mathrm{Te}_{3}$ thin film and the $\mathrm{Si}$ substrate with a native $\mathrm{SiO}_{2}$ layer $(-2.73 \mathrm{~nm})$. The detailed theoretical calculation of the reflection and the phase shift can be found in Supplementary Note 1. The optical parameters for the simulation of the $\mathrm{Sb}_{2} \mathrm{Te}_{3}$ thin film can be obtained from Fig. $2 b, c$ and the parameters for $\mathrm{Si}$ are obtained from the reference ${ }^{25}$. Figure $3 \mathrm{a}$ shows the simulated distributions of electromagnetic fields in the resonant cavity under illumination of the $632 \mathrm{~nm}$ laser beam and the electromagnetic fields uniformly distribute on the Si substrate without the $\mathrm{Sb}_{2} \mathrm{Te}_{3}$ thin film for comparison. Distinct enhancement of electromagnetic fields can be noticed in the resonant cavity.

The simulated phase shift (between the $60 \mathrm{~nm} \mathrm{Sb}_{2} \mathrm{Te}_{3}$ thin films and the $\mathrm{SiO}_{2} / \mathrm{Si}$ substrate) as a function of the film thickness at the wavelengths 445,532 and $632 \mathrm{~nm}$ light are shown in Fig. $3 \mathrm{~b}$. It is obvious that the phase shift monotonically increases when the thickness of the $\mathrm{Sb}_{2} \mathrm{Te}_{3}$ thin films increases. The phase 
a

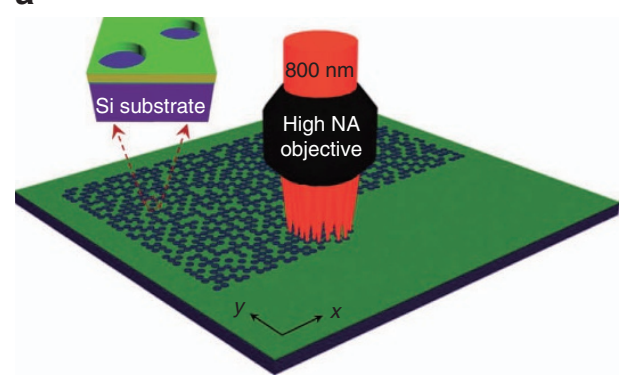

b

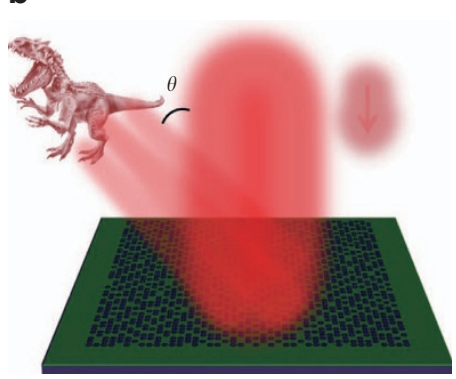

Figure 1 | Nanofabrication of the binary hologram and holographic imaging procedures. (a) Nanofabrication process of a nanometric $\mathrm{Sb}_{2} \mathrm{Te}_{3}$ hologram using the direct laser write system. The upper inset displays the ablated pixels in the topological insulator thin film. (b) Holographic imaging procedure of a nanometric $\mathrm{Sb}_{2} \mathrm{Te}_{3}$ hologram using a continuous wavelaser beam. $\theta$ is the projection angle of the holographic image. Note: this figure is not included under the article CC BY licence; Indominus Rex image is reproduced with permission from the publisher Comingsoon.net and copyright owner Universal Studios.

\section{a}

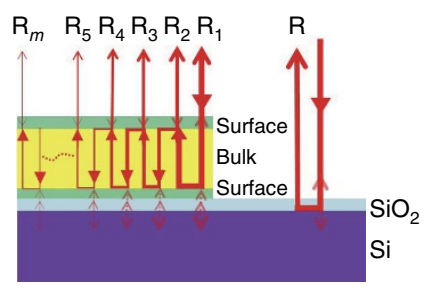

b

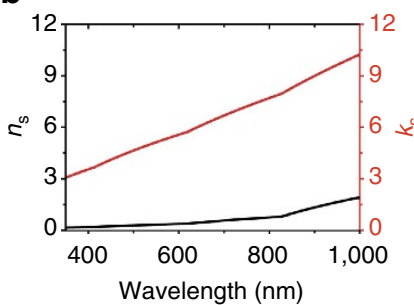

C

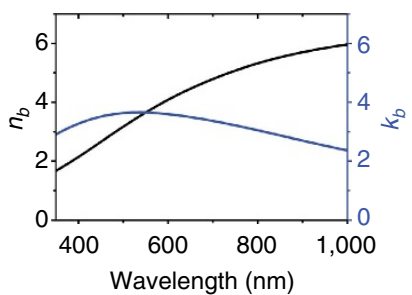

Figure 2 | Physical mechanism of the $\mathbf{S b}_{\mathbf{2}} \mathbf{T} \mathbf{e}_{\mathbf{3}}$ thin film cavity. (a) Diagram of internal light multiple reflections in the resonant cavity of the $\mathrm{Sb}_{2} \mathrm{Te}_{3}$ thin film. The semi-transparent arrows indicate interface reflections. (b) The surface refractive index $n$ and extinction coefficient $k$ curves of the $\mathrm{Sb}_{2} \mathrm{Te}_{3}$ thin film as a function of wavelengths. (c) The bulk refractive index $n$ and extinction coefficient $k$ curves of the $\mathrm{Sb}_{2} \mathrm{Te}_{3}$ thin film as a function of wavelengths.

a

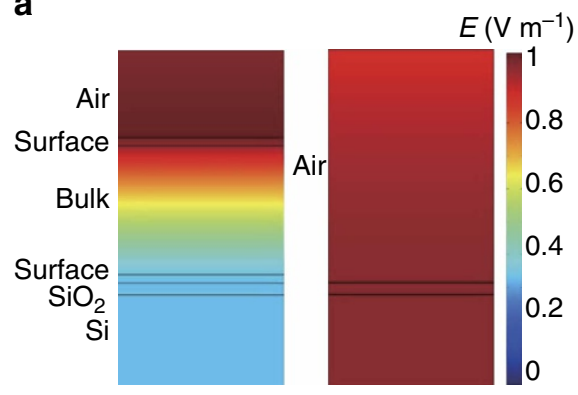

c

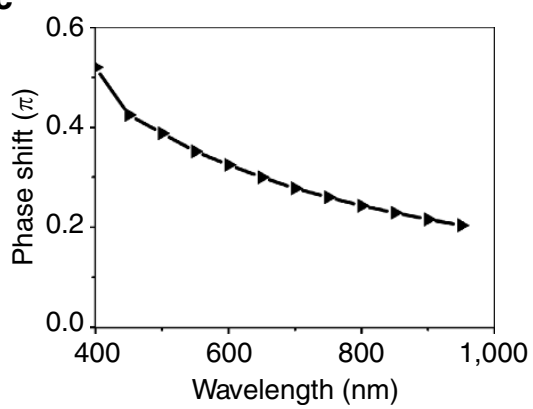

b

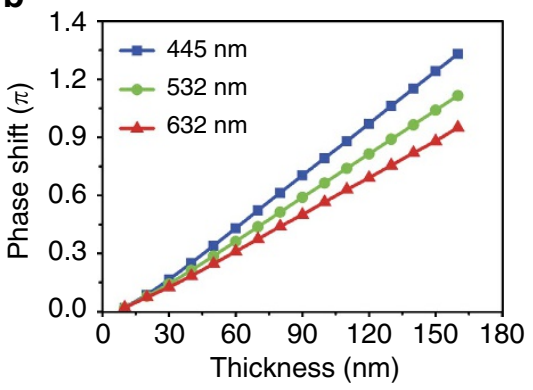

d

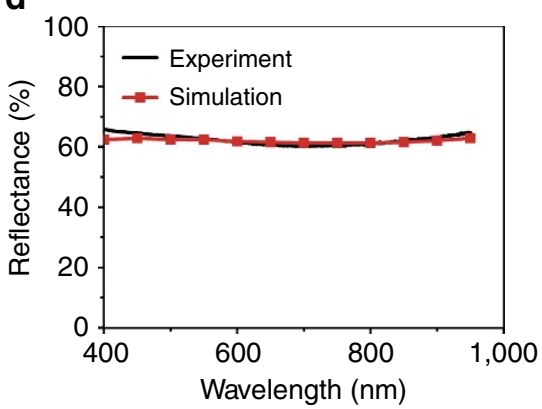

Figure 3 | Numerical simulations of electromagnetic fields and phase shift. (a) Simulated electromagnetic field distributions in the $\mathrm{Sb}_{2} \mathrm{Te}_{3}$ thin film cavity and the $\mathrm{SiO}_{2} / \mathrm{Si}$ substrate under illumination with $632 \mathrm{~nm}$ light. (b) Simulated phase shift between the $\mathrm{Sb}_{2} \mathrm{Te}_{3}$ film and the $\mathrm{SiO}_{2} / \mathrm{Si}$ substrate as a function

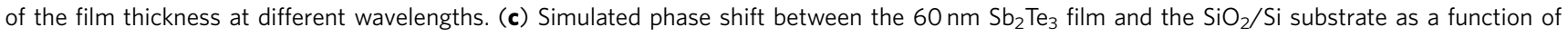
wavelength. (d) Simulated and experimental reflectance of $60 \mathrm{~nm} \mathrm{Sb}{ }_{2} \mathrm{Te}_{3}$ thin films as a function of wavelength. The simulation shows good agreement with experiments.

shift is $\sim 0.5 \pi, 0.4 \pi$ and $0.3 \pi$ at the wavelengths 445,532 and $632 \mathrm{~nm}$, respectively. Figure $3 \mathrm{c}$ shows the simulated phase shift between the $\mathrm{Sb}_{2} \mathrm{Te}_{3}$ thin film and $\mathrm{SiO}_{2} / \mathrm{Si}$ as a function of wavelength. It can be found that the phase shift decreases when the wavelength increases. These results demonstrate that $\mathrm{Sb}_{2} \mathrm{Te}_{3}$ thin films generate higher quality holographic images with shorter wavelengths. Figure $3 \mathrm{~d}$ demonstrates the simulated and experimental reflectance of the $60 \mathrm{~nm} \mathrm{Sb}_{2} \mathrm{Te}_{3}$ thin film as a 
function of wavelength. It can be found that the simulated reflectance agrees with experimental results very well. The simulated reflectance as a function of thin film thickness can be found in Supplementary Fig. 3a, and the reflectance of substrate can be found in Supplementary Fig. $3 b$.

Optical characterization of nanometric holograms. $\mathrm{Sb}_{2} \mathrm{Te}_{3}$ has been both theoretically and experimentally confirmed as a topological insulator material ${ }^{26,27}$. In this work, the $\mathrm{Sb}_{2} \mathrm{Te}_{3}$ thin films are grown on Si wafers by using an atomic layer deposition (ALD) system. The ALD method is capable of growing nanometric films layer by layer on a large scale and at a relatively low temperature. The growth details of the $\mathrm{Sb}_{2} \mathrm{Te}_{3}$ thin films can be found in the Methods.

Figure $4 \mathrm{~b}, \mathrm{c}$ show the scanning electron microscope (SEM) images of laser written hologram patterns on the $\mathrm{Sb}_{2} \mathrm{Te}_{3}$ thin films. With fslaser ablation, the nanometric $\mathrm{Sb}_{2} \mathrm{Te}_{3}$ film is completely removed and the native $\mathrm{SiO}_{2}$ film is exposed to air. The $\mathrm{Sb}_{2} \mathrm{Te}_{3}$ film in the area without laser ablation results in a strong phase modulation due to multiple reflections in the optical cavity. However, the native $\mathrm{SiO}_{2}$ layer generates a weak phase modulation due to its small refractive index. Thus, two levels of phase modulations can be generated, leading to the formation of binary holograms.

The holographic images can be generated through the reconstruction of wavefronts of light with the phase information. By illuminating a laser beam on the surface of the topological insulator hologram, the holographic images are diffracted from it with a small projection angle of $10^{\circ}$. A full colour CCD was used to capture the reconstructed images. The laser beam power and its focal position are adjusted to obtain the optimal imaging quality. Figure $4 \mathrm{a}$ displays the original picture of dinosaur object. Figure $4 \mathrm{~d}-\mathrm{f}$ show the holographic images of the dinosaur from the $60 \mathrm{~nm}$-thick hologram at different wavelengths. The signal-tonoise ratio, which is defined as the ratio of the peak intensity in the reconstructed image to the s.d. of the background noise ${ }^{10}$, can be utilized to evaluate the quality of the reconstructed image. The background noise is taken from an area of $120 \times 120$ pixels beside the image. The signal-to-noise ratios for the reconstructed images from Fig. $4 \mathrm{~d}-\mathrm{f}$ are 39, 33 and 29, respectively. The reconstructed images demonstrate a higher quality at shorter wavelengths due to the larger phase modulation.
The measured conversion efficiency of the $60 \mathrm{~nm}$-thick hologram is approximately $1.6,1.5$ and $1.35 \%$ at the wavelengths 445,532 and $632 \mathrm{~nm}$, respectively. The conversion efficiency is defined as the ratio of the power of the holographic image and the input power. In comparison, the efficiency of topological insulator holograms is lower than dielectric metasurface holograms. One main reason is that the hologram we have used is a binary one which causes more noises than multiple-level holograms. Another reason is that the phase shift for a $60 \mathrm{~nm}$ hologram $(0-\pi / 2)$ is still smaller than a metasurface modulated phase shift $(0-2 \pi)$. Through increasing the thickness and the phase modulation levels, the topological insulator hologram efficiency can be further improved. In addition, the light absorption in the $\mathrm{Sb}_{2} \mathrm{Te}_{3}$ thin film is higher than dielectric metasurface in the visible range. The efficiency of topological insulator holograms can be improved through using highly insulating topological insulator materials with a higher bulk refractive index.

\section{Discussion}

The performance of the nanometric holograms is determined by several parameters. First, in the nanofabrication process, the power density of the fs-laser beam affects the hologram morphology and resolution. In our experiments, the laser beam fluence of $0.1 \mathrm{~J} \mathrm{~cm}^{-2}$ is optimal for obtaining high quality hologram patterns and holographic images. Second, the thickness of the $\mathrm{Sb}_{2} \mathrm{Te}_{3}$ thin films plays a key role in the quality and efficiency of holographic images. In principle, the thicker holograms generate a larger phase shift and thus higher quality holographic images. Furthermore, the images noise is lower for thicker holograms. These can be found through comparing the $60 \mathrm{~nm}$ thick hologram with a $25 \mathrm{~nm}$-thick hologram in Supplementary Fig. 4c. Third, in the holographic imaging process, the laser wavelengths affect the holographic images and the hologram efficiency. We have found that the shorter wavelength produces the higher efficiency due to the larger phase shift. However, the light absorption is enhanced in the ultraviolet frequency range, which limits the further improvement of the hologram quality and efficiency. Therefore, to achieve the best holographic image, these parameters need to be balanced.

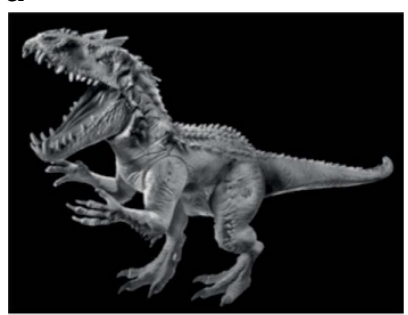

d

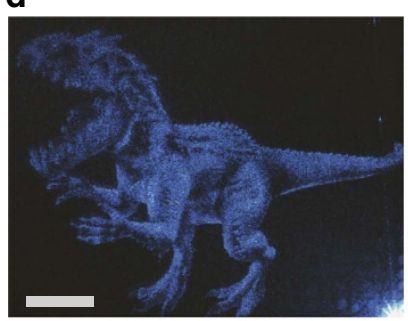

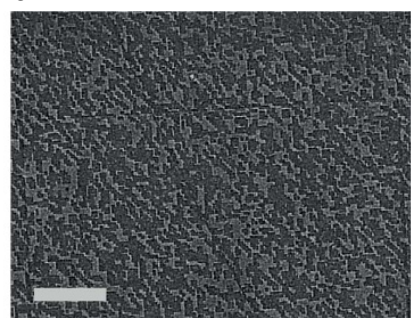

e

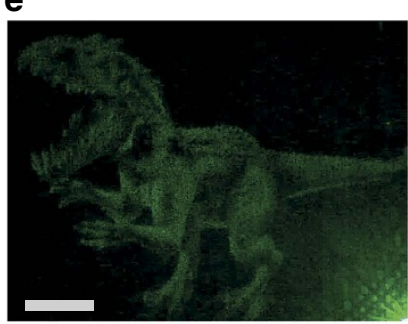

C

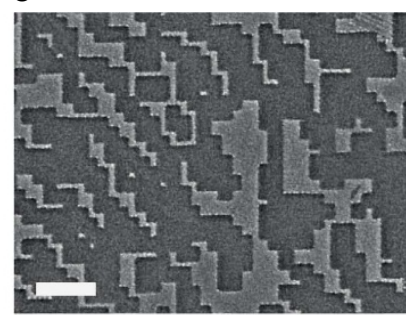

f

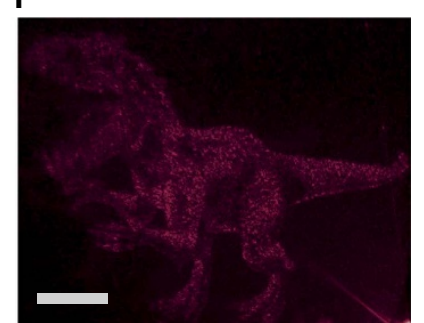

Figure 4 | Nanometric $\mathbf{S b}_{\mathbf{2}} \mathrm{Te}_{\mathbf{3}}$ thin film holograms and reconstructed images. (a) Original image of the dinosaur object. Note: this figure is not included under the article CC BY licence; Indominus Rex image is reproduced with permission from the publisher Comingsoon.net and copyright owner Universal Studios. (b,c) SEM images of the laser printed hologram patterns. The scale bar is $50 \mu \mathrm{m}$ for $\mathbf{b}$ and $10 \mu \mathrm{m}$ for $\mathbf{c}$, respectively. (d-f) Holographic images captured by illuminating the nanometric holograms using 445, 532 and $632 \mathrm{~nm}$ continuous wavelaser beams. Scale bars for $\mathbf{d}-\mathbf{f}$ are $1 \mathrm{~mm}$. 
Compared with the fabrication methods of metasurface holograms, the DLW method does not require the complicated substrate treatment and the mask preparation process. Hence, the DLW fabricated nanometric holograms are more suitable for large-scale practical applications. They are promising to be integrated into flat electronic devices for optical imaging, data storage and security. They can also be used as anti-fake stamps for credit cards and currencies. In addition, with the unique function of enhancing phase shifts, a topological insulator cavity can also be used to design nanometric photo-detectors and lenses.

\section{Methods}

ALD growth of $\mathbf{S b}_{2} \mathbf{T e}_{3}$ thin films. The chemical equation for synthesizing $\mathrm{Sb}_{2} \mathrm{Te}_{3}$ is $\mathrm{Sb}\left(\mathrm{OC}_{2} \mathrm{H}_{5}\right)_{3}+3\left[\left(\mathrm{CH}_{3}\right)_{3} \mathrm{Si}\right]_{2} \mathrm{Te} \rightarrow \mathrm{Sb}_{2} \mathrm{Te}_{3}+6\left(\mathrm{CH}_{3}\right)_{3} \mathrm{Si}-\mathrm{OC}_{2} \mathrm{H}_{5}$ (ref. 28). The injection/ purge times for the precursors of $\mathrm{Sb}\left(\mathrm{OC}_{2} \mathrm{H}_{5}\right)_{3}$ and $3\left[\left(\mathrm{CH}_{3}\right)_{3} \mathrm{Si}\right]_{2} \mathrm{Te}$ were $0.75 \mathrm{~s} / 9 \mathrm{~s}$ and $0.1 \mathrm{~s} / 9 \mathrm{~s}$, respectively. As $\mathrm{Sb}\left(\mathrm{OC}_{2} \mathrm{H}_{5}\right)_{3}$ was difficult to gasify, an Ar gas booster was used on the cylinder to promote the gasification process. The precursor transport temperature was kept at $150^{\circ} \mathrm{C}$. The substrate temperature in the deposition process was kept at $70^{\circ} \mathrm{C}$, which was optimal for a maximum growth rate. The chamber vacuum was $2 \times 10^{-2}$ Torr. The growth rate was $0.01 \mathrm{~nm}$ per cycle.

The X-ray diffraction patterns (Supplementary Fig. 2a) identify that the $\mathrm{Sb}_{2} \mathrm{Te}_{3}$ thin film is highly crystalline and grown along the $c$ axis. The Raman spectrum (Supplementary Fig. 2b) confirms that the composition of the thin film is $\mathrm{Sb}_{2} \mathrm{Te}_{3}$. The Atomic Force Microscopy (Supplementary Fig. 2c) demonstrates the surface morphology of the thin films. The measured bulk band gap of the $\mathrm{Sb}_{2} \mathrm{Te}_{3}$ thin films is $0.15 \mathrm{eV}$ (Supplementary Fig. 3c). These characterizations reveal the high quality of the ALD grown $\mathrm{Sb}_{2} \mathrm{Te}_{3}$ thin film.

Direct Laser writing system. A femtosecond-pulsed laser beam at a wavelength of $800 \mathrm{~nm}$ (Maitai, repetition rate: $10 \mathrm{KHz}$ and pulse width: $100 \mathrm{fs}$ ) was employed for the writing of hologram patterns. An objective with numerical aperture of 0.7 was used to focus the pulsed laser beam onto the surface of the $\mathrm{Sb}_{2} \mathrm{Te}_{3}$ thin film. The average laser beam fluence was adjusted by an ND filter and was kept at $0.1 \mathrm{Jcm}^{-2}$ in the fabricating process. The holographic patterns were achieved by laterally translating the $3 \mathrm{D}$ translation stage across the focal plane.

Optical measurements. The refractive index and extinction coefficient were measured using a multiple-angle spectroscopic ellipsometer (J.A. Woollam Co. $\mathrm{M}-2000)$. The thickness of the $\mathrm{Sb}_{2} \mathrm{Te}_{3}$ thin film was determined through fitting the data from ellipsometer measurements. The thickness of the surface layer was $1.5 \mathrm{~nm}$, and the thickness remains constant when the total thickness of the thin film increases. The reflection spectroscopy was measured using an ultraviolet-visible spectrophotometer.

Data availability. All data used to obtain the conclusions in this paper are available in the paper and/or the Supplementary Information. Other data may be requested from the authors.

\section{References}

1. Gabor, D. A new microscopic principle. Nature 161, 777-778 (1948).

2. Li, X. et al. Athermally photoreduced graphene oxides for three-dimensional holographic images. Nat. Commun. 6, 6984 (2015).

3. Ye, W. et al. Spin and wavelength multiplexed nonlinear metasurface holography. Nat. Commun. 7, 11930 (2016).

4. Brown, B. R. \& Lohmann, A. W. Complex spatial filtering with binary masks. Appl. Opt. 5, 967-969 (1966).

5. Slinger, C., Cameron, C. \& Stanley, M. Computer-generated holography as a generic display technology. Computer 38, 46-53 (2005).

6. Xue, G. et al. Multiplexing encoding method for full-color dynamic 3D holographic display. Opt. Express 22, 18473-18482 (2014).

7. Yu, N. \& Capasso, F. Flat optics with designer metasurfaces. Nat. Mater. 13, 139-150 (2014)

8. Kildishev, A. V., Boltasseva, A. \& Shalaev, V. M. Planar photonics with metasurfaces. Science 339, 1232009 (2013).

9. Ren, H., Li, X., Zhang, Q. \& Gu, M. On-chip noninterference angular momentum multiplexing of broadband light. Science 352, 805-809 (2016).

10. Ni, X. J., Kildishev, A. V. \& Shalaev, V. M. Metasurface holograms for visible light. Nat. Commun. 4, 2807 (2013)

11. Huang, L. et al. Three-dimensional optical holography using a plasmonic metasurface. Nat. Commun. 4, 2808 (2013).

12. Zheng, G. et al. Metasurface holograms reaching $80 \%$ efficiency. Nat. Nanotechnol. 10, 308-312 (2015).

13. Khorasaninejad, M., Ambrosio, A., Kanhaiya, P. \& Capasso, F. Broadband and chiral binary dielectric meta-holograms. Sci. Adv. 2, e1501258 (2016).

14. Bonaccorso, F., Sun, Z., Hasan, T. \& Ferrari, A. C. Graphene photonics and optoelectronics. Nat. Photon. 4, 611-622 (2010).
15. Wang, Q. H., Kalantar-Zadeh, K., Kis, A., Coleman, J. N. \& Strano, M. S Electronics and optoelectronics of two-dimensional transition metal dichalcogenides. Nat. Nanotechnol. 7, 699-712 (2012).

16. Hasan, M. Z. \& Kane, C. L. Colloquium: topological insulators. Rev. Mod. Phys. 82, 3045-3067 (2010).

17. Qi, X.-L. \& Zhang, S.-C. Topological insulators and superconductors. Rev. Mod. Phys. 83, 1057-1110 (2011).

18. Peng, H. et al. Topological insulator nanostructures for near-infrared transparent flexible electrodes. Nat. Chem. 4, 281-286 (2012).

19. Di Pietro, P. et al. Observation of Dirac plasmons in a topological insulator. Nat. Nanotechnol. 8, 556-560 (2013).

20. Ou, J.-Y. et al. Ultraviolet and visible range plasmonics in the topological insulator $\mathrm{Bi}_{1.5} \mathrm{Sb}_{0.5} \mathrm{Te}_{1.8} \mathrm{Se}_{1,2}$. Nat. Commun. 5, 5139 (2014).

21. Yue, Z., Cai, B., Wang, L., Wang, X. \& Gu, M. Intrinsically core-shell plasmonic dielectric nanostructures with ultrahigh refractive index. Sci. Adv. 2, e1501536 (2016).

22. Lin, H., Jia, B. \& Gu, M. Dynamic generation of Debye diffraction-limited multifocal arrays for direct laser printing nanofabrication. Opt. Lett. 36, 406-408 (2011).

23. Gu, M. Advanced Optical Imaging Theory (Springer Science \& Business Media, 2000).

24. Kuwahara, M. et al. Approach for measuring complex refractive index of molten Sb2Te3 by spectroscopic ellipsometry. Appl. Phys. Lett. 100, 101910 (2012).

25. Green, M. A. \& Keevers, M. J. Optical properties of intrinsic silicon at $300 \mathrm{~K}$. Prog. Photovolt. 3, 189-192 (1995)

26. Zhang, H. et al. Topological insulators in $\mathrm{Bi}_{2} \mathrm{Se}_{3}, \mathrm{Bi}_{2} \mathrm{Te}_{3}$ and $\mathrm{Sb}_{2} \mathrm{Te}_{3}$ with a single Dirac cone on the surface. Nat. Phys. 5, 438-442 (2009).

27. Zhang, J. et al. Band structure engineering in $\left(\mathrm{Bi}_{1-x} \mathrm{Sb}_{x}\right)_{2} \mathrm{Te}_{3}$ ternary topological insulators. Nat. Commun. 2, 574 (2011).

28. Eom, T. et al. Conformal formation of $\left(\mathrm{GeTe}_{2}\right)(1-x)\left(\mathrm{Sb}_{2} \mathrm{Te}_{3}\right)_{x}$ layers by atomic layer deposition for nanoscale phase change memories. Chem. Mater. 24, 2099-2110 (2012).

\section{Acknowledgements}

M.G. acknowledges the support from the Australian Research Council (ARC) through the Discovery Project (DP140100849). Y.W. and J.L. acknowledge the National Natural Science Founding of China (61420106014) and the National Basic Research Program of China (973 Program Grant No. 2013CB328801 and No. 2013CB328806). We thank Drs Lachlan Hyde, Deming Zhu and Xiaohan Yang for technical assistances in ALD growth, Raman and ultraviolet-visible spectrophotometer measurements, respectively. We thank Drs Qiming Zhang and Haoran Ren for helpful discussions. This work was performed in part at the Centre for Micro-Photonics in Swinburne University of Technology and the Melbourne Centre for Nanofabrication (MCN) in the Victorian Node of the Australian National Fabrication Facility (ANFF).

\section{Author contributions}

M.G. and Z.Y. conceived the idea and design the experiments. Z.Y. prepared and characterized the materials. G.X. calculated the hologram and conducted the theoretical computation. Z.Y. and G.X. fabricated the holograms and captured the holographic images. G.X. and Z.Y. conducted the Comsol and Matlab simulation. All authors contributed to the data analysis, manuscript writing and discussion.

\section{Additional information}

Supplementary Information accompanies this paper at http://www.nature.com/ naturecommunications

Competing interests: The authors declare no competing financial interests.

Reprints and permission information is available online at http://npg.nature.com/ reprintsandpermissions/

How to cite this article: Yue, Z. et al. Nanometric holograms based on a topologica insulator material. Nat. Commun. 8, 15354 doi: 10.1038/ncomms15354 (2017).

Publisher's note: Springer Nature remains neutral with regard to jurisdictional claims in published maps and institutional affiliations.

This work is licensed under a Creative Commons Attribution 4.0 International License. The images or other third party material in this article are included in the article's Creative Commons license, unless indicated otherwise in the credit line; if the material is not included under the Creative Commons license users will need to obtain permission from the license holder to reproduce the material. To view a copy of this license, visit http://creativecommons.org/licenses/by/4.0/

(C) The Author(s) 2017 